\title{
La Pedagogía de la Respuesta como modelo pedagógico La formación humanista Tomasiana-Sedaniana
}

Fr. Wilson Fernando MENDOZA RIVERA, O.P. '

Recibido: 10-09-2019

Aceptado: 02-11-2019

\section{Introducción}

El título del presente artículo está inspirado en el pensamiento dominicano y tomista del sacerdote colombiano, fray José de Jesús Sedano González, O.P., y en su reflexión La Pedagogía de la Respuesta. El punto de partida es preguntarse si la pedagogía de la respuesta ¿Es O no un modelo pedagógico? Para responder a la pregunta planteada es importante tener presente las siguientes consideraciones: De manera general, aplicar los criterios de todo modelo pedagógico a la pedagogía de la respuesta para conocer el tipo de hombre y de sociedad que se quieren contribuir a formar. De manera particular, describir las teorías fundamentales y com-

\footnotetext{
1. Bachiller en Sagrada Teología - Pontificia Universidad Bolivariana (Medellín-Colombia). Licenciado en filosofía y Cultura para la Paz - Universidad Santo Tomás, Abierta y a Distancia - VUAD (Bogotá-Colombia). Licenciado en Teología - Universidad Santo Tomás, Abierta y a Distancia - VUAD (Bogotá-Colombia ). Magister en Estudios Dominicanos - Universidad de Salamanca (Salamanca - España). Magister en pedagogía - Universidad Santo Tomás (TunjaColombia). Magister en Estudios Humanisticos y Sociales - Universidad Abat Oliba - Centro de Estudios Universitarios (Barcelona - España). Doctor en Estudios Tomísticos - Universidad Abat Oliba - Centro de Estudios Universitarios (Barcelona - España). Posdoctor en Educación - Universidad Santo Tomas (Bogota - Colombia).

Docente de la Universidad San Buenaventura (Cali). Docente de la Universidad Santo Tomás (Tunja). Docente de la Facultad de Filosofía de la USTA - Bogotá. Docente de la Facultad de Teología USTA- Bogotá. Docente en el Colegio de Jordán de Sajonia (Bogotá). Docente en el Colegio Santo Tomás de Aquino (Bogotá). Rector del Colegio Santo Tomás de Aquino (Bogotá)
} 
ponentes curriculares que orientan la propuesta pedagógica de la pedagogía de la respuesta; finalmente, interpretar la propuesta pedagógica de la pedagogía de la respuesta como horizonte de conceptualización para proponer un modelo de pedagogía humanista.

Para el desarrollo de la pregunta planteada se enuncia la siguiente hipótesis: La pedagogía de la respuesta es un modelo pedagógico porque reúne los fundamentos y componentes de toda propuesta pedagógica, pero al mismo tiempo puede ser un modelo de pedagogía humanista por estar fundamentada en el pensamiento dominicano y tomista. La metodología utilizada es la teoría fundamentada para el desarrollo de una nueva teoría pedagógica. De modo que la presente investigación

se circunscribe en el paradigma investigativo cualitativo de tipo teoría fundamentada que, a su vez, posibilita comparar y contrastar la teoría existente y la construida durante la investigación. De manera que, la lectura de la pedagogía de la respuesta, en el marco del modelo pedagógico, nos permite construir una nueva teoría pedagogica que vamos a llamar la pedagogía humanista.

\section{Cuestiones preliminares}

El contexto en que fray José de Jesús Sedano pensó y escribió su propuesta pedagógica, es decir, la "Pedagogía de la Respuesta", se encuadra en la tradición de la formación religiosa dominicana que recibió y en su estudio del pensamiento de Santo Tomás de Aquino. Fray José de Jesús Sedano Gonzáles nació en Vélez, Santander, el 25 de febrero de 1922. Fray Sedano perteneció a la comunidad religiosa Orden de Predicadores de la Provincia San Luís Bertrán de Colombia en la que profesó el 2 de enero de 1940, y se ordenó sacerdote el 5 de enero de 1947 en Bogotá. Murió el 3 de enero de 2019, a los 97 años de edad en el Convento de Cristo Rey de Bucaramanga (Santander). Como formador o maestro no creó una escuela que pudiéramos Ilamar "la sedanidad", pero sí dejó una impronta pedagógica: no me des las respuestas, enséñame a responder. 
La persona que más ha incidido en la formación personal y dominicana de fray Sedano fue su maestro de novicios fray Gabriel María Blanchet. Al maestro Blanchet:

Ante todo, le importaba que sus novicios pudiesen "mirar con la mirada de Cristo y de sto. Domingo" y que adquiriesen un verdadero criterio teológico y sobrenatural para poder así llegar a ser verdaderamente "autónomos", esto es, capaces de obrar por convicción personal, siguiendo una regla interior, sin necesidad de que se le estuviese vigilando (Blanchet, p. 2).

El maestro francés, fray Blachet, señala sobre la formación de la persona, en la etapa de vida religiosa llamada noviciado, lo siguiente:

Por eso es necesario tratarlo como al germen de la planta o, mejor, del ser, del hombre que deberá ser más tarde en plenitud. No es germen para serlo siempre; es germen pero de algo. Debe tener continuidad de vida, en los diferentes estadios, entre lo que es y lo que será más tarde (Blanchet, p. 16).

Estas consideraciones sobre la formación de la persona del maestro Blanchet fueron forjando la personalidad de fray Sedano porque las desarrollo en su vida y en los contextos educativos como maestro. Las notas que caracterizan la personalidad del maestro fray Sedano son las de formador, la de tomista y humanista. Como maestro enseñó a responder antes que dar respuesta: responder de sí mismo, por sí mismo. Gran parte de su vida la ha dedicado a la formación de religiosos y de laicos en los centros de formación dominicana, especialmente en el Colegio Jordán de Sajonia y en la Universidad Santo Tomás. Fray Sedano implementa la pedagogía de la respuesta en el Seminario Apostólico (hoy Colegio) Jordán de Sajonia, donde llega a ser rector en 1954, y donde formó para la democracia, es decir, para la capacidad de decidir y para el bien común, que ha quedado plasmado en el lema del escudo del Colegio Jordán de Sajonia como: Stemus simul (Cárdenas, 2019, p. 21). 
Por otra parte, el maestro fray Sedano ha sido un estudioso del pensamiento tomista: "Su dedicación a Santo Tomás la estimuló en él sobre todo el padre Tomás María Quijano" (Correa, 2019, p. 12). De igual manera, la lectura asídua de la obra de Santo Tomás de Aquino concibió en la mente de fray Sedano una filosofía de la formación basada en el realismo tomasiano, cuyo enfoque personista y social quedará plasmado en su reflexión pedagógica. Su estancia académica en la Universidad Santo Tomás de Roma, También conocida como el Angélico, consolidó su conocimiento en el Tomismo. En su tesis doctoral sobre el don del consejo, como don del Espíritu Santo y como parte potencial o aneja a la virtud moral de la prudencia en Tomás de Aquino, deja en evidencia su conocimiento en el pensamiento tomasiano.

Finalmente, su humanismo se centra en la educación de la persona a quien siempre ha considerado en potencia o como una semilla -de humanidad- Ilamada a crecer para dar lo mejor de sí. (Cárdenas, 2019, p. 19).

El contexto religioso, dominicano y tomista en el que se ha desarrollado la propuesta pedagógica de fray Sedano podría parecer una limitante, para elaborar un modelo pedagógico, no obstante, susceptible, al decir del autor, de convertirse en marco doctrinal para un proyecto educativo $y$, de esta manera, repensar la formación de la persona entre los diferentes modelos pedagógicos existentes (Sedano, 2017, p. 25).

En la actualidad, los modelos pedagógicos vigentes son diversos y diferentes. Entre ellos podemos mencionar los siguientes: el modelo pedagógico instruccionista, el pedagógico activista, el pedagógico constructivista, el pedagógico crítico, el pedagógico dialogante, el pedagógico liberador y el pedagógico holistico transformador. Todos ellos tienen en común la finalidad del acto educativo, a saber: responder el interrogante en torno al tipo de hombre y sociedad que se quiere contribuir a formar (De Zubiría, 2015, p. 41).

La propuesta pedagógica de fray Sedano, se ha construído y consolidado a través de un proceso depurativo y significativo. En 
él, desde sus inicios, no pretendió escribir un tratado de pedagogía con miras a pensarse como modelo pedagógico. Su propuesta central ha sido la pedagogía de la respuesta. La primera versión que se publicó bajo este título fue: Hacia una Pedagogía de la Respuesta. Horizonte dominicano para la formación de la persona (1996). Como el subtítulo lo indica, el texto está orientado a la formación de la persona en la vida religiosa dominicana, en el contexto propio de la Orden de Predicadores en Colombia. La segunda versión se publicó con el titulo: Pedagogía de la Respuesta. Horizontes para la formación humana (2002). En este caso la obra se extiende al ámbito secular $y$, por lo tanto, a otro público fuera del religioso. Finalmente, una tercera versión orientada a un ambiente propiamente académico y universitario, con un interés en la educación integral de la persona desde una perspectiva tomista, aparece con el título: Hacía una pedagogía de la respuesta. Horizonte tomasino para la formación integral de la persona (2012). En sus tres ediciones es notorio el componente dominicano y tomista en el que fue formado fray Sedano, así como su experiencia de vida y de formación de religiosos y laicos. De modo que en todas ellas, hay una constante categorial que subyace, a saber: dominicano, tomista, formador, humanista.

\section{La teoría pedagógica de la pedagogía de la respuesta}

¿Cuáles son los fundamentos que constituyen un modelo pedagógico? Al decir de Zubiría (1994, p. 39) el modelo pedagógico se fundamenta y se construye sobre una teoría pedagógica, que a su vez está elaborada por una teoría psicológica, una teoría social y una teoría antropológica.

Los fundamentos del modelo pedagógico -sin detrimento de lo señalado por Zubiría sino apoyados en él- se pueden ordenar de la siguiente manera: el fundamento antropológico que responde la pregunta ¿Qué es el hombre?; el fundamento epistemológico que responde la pregunta ¿Cómo conoce el hombre?; el fundamento 
metodológico que responde a la pregunta ¿De qué manera se debe enseñar? Las respuestas a estas preguntas nos conducen a la construcción de la teoría pedagógica que reflexiona sobre el fin del acto educativo.

\subsection{Fundamento antropológico de la pedagogía de la respuesta}

¿Qué es el hombre? El fundamento antropológico de la pedagogía de la respuesta se inspira en la estructura antropológica de Santo Tomás de Aquino. Para el Aquinate el hombre es una realidad integral (Summa Theol., I q. 75 art. 4 Sed Cont), que actúa por medio de unas facultades o potencias (Summa Theol., I q. 77 art. 6 Sed Cont). De igual modo, el hombre tiene una variedad de potencias por hallarse en el horizonte entre las creaturas espirituales y corporales (Summa Theol., I q. 77 art. 2), es decir, el hombre es un microcosmos de potencialidades. Aunque Santo Tomás establece una distinción entre las potencias propias del alma (entendimiento) como del compuesto (vegetativa, locomotriz, sensitiva, apetitiva) afirma que todas ellas dimanan del alma como de su principio (Summa Theol., I q. 77 art. 3). Las potencias o facultades se activan, se desarrollan y se perfeccionan a través de los actos (Summa Theol., I q. 79 art. 2).

La antropovisión de Santo Tomás, va a incidir en el desarrollo antropológico de fray Sedano. De esta manera, para él, el hombre es una realidad total pero no acabada: "Entonces empezará a comprender que su vida, su vida intrasferible, es un "ya es" y un "todavía no es", porque es un ser que ha de realizarse (Sedano, 2017, p.43). De modo que fray Sedano desarrolla los conceptos de la antropología tomasiana de potencia y acto, aunque más del concepto acto en su estudio y concepción sobre el hombre. De manera que, el hombre es una realidad dinámica: es y se hace en la medida que pone en actos sus facultades naturales.

Fray Sedano para explicar su concepción de hombre, como una realidad dinámica, hace uso de las categorías de germen, proyecto 
y realización (2017, p. 44). De esta manera, el hombre está llamado a ser, haciéndose, es decir, pasando de la potencia al acto. Además, en el pensamiento de fray Sedano es importante subrayar la dimensión teologal del hombre en su proceso de formación desde la perspectiva de la antropología teológica. El hombre se semejanza de Dios que es acto puro, en la medida que pone en acto sus capacidades. En el hombre anida el deseo natural de su plena realización.

Ciertamente, sólo Dios posee la absoluta plenitud de su ser; "es acto puro", "actualidad purísima", omnímodamente bueno porque nada de su ser le queda por realizar; siempre y desde siempre es la realización de su existencia. En cambio, el ser del hombre, de todo hombre, de todos los hombres, como el de toda realidad creada, es un potencial vivo y dinámico con apetito de realización plena (Sedano, 2017, p. 60).

Del mismo modo, la felicidad del hombre y su fin último que es Dios, consiste en su realización como persona. Así, para Fray Sedano (2017): "Es decir, que mi bien o mi mal, mi felicidad o mi infelicidad está en razón directa con mi realización o mí no-realización" (p.50). Por tanto, para él, el hombre, es una semilla en crecimiento y desarrollo. El hombre es y se hace en la medida que ponga en acto sus potencias o facultades. Así lo señala la experiencia sajoniana: "El hombre tiende a desarrollarse, crecer y ser productivo. La parálisis de esa tendencia es sintomática de enfermedad" (1975, p. 105).

Además, fray Sedano asume del pensamiento Tomasiano la idea del hombre como un ser personal y social. El hombre es persona, es deicir, un ser individual y al mismo tiempo social por naturale$z a$. De manera que, fray Sedano retoma tres aspectos importantes del hombre, desde la perspectiva de Santo Tomás de Aquino: la persona, la fragilidad y la comunicación. Para Tomás de Aquino y en seguimiento de Aristóteles, "corresponde a la naturaleza del hombre ser un animal sociable y político" (De regim. princ., lib.1). Además, "que vive sociedad, más aún que el resto de los animales, 
cosa que nos revela su misma necesidad natural" (Cont. Gentes., lib. 1). Pues el hombre al nacer, a diferencia del resto de los animales, es el más frágil y vulnerable como nos lo revela su complexión corporal (Summa Theol., I q. 91 art. 3 ad 2). Así, la misma condición natural del hombre, frágil y vulnerable lo hace sociable, porque en sociedad suple sus necesidades físicas y corporales. De modo que, el hombre se realiza como persona en sociedad.

El Aquinate subraya que el hombre, en lugar de todas aquellas cosas que la naturaleza ha dotado a los demás animales como la velocidad, dientes, cuernos garras, se le ha dado la razón y las manos de las que se puede servir de muchas maneras para vivir y atender sus necesidades (Cont. Gentes., lib. 1). Sin embargo, a pesar de que el hombre pueda abastecer en gran parte sus necesidades a través de la razón y de las manos, sigue siendo un ser social porque si fuera un ser que se bastara por sí mismo o fuera autosuficiente, sería un dios o una bestia (Summa Theol., II-II q. 188 art. 8 ad 5).

Además, una de las dimensiones fundamentales del hombre por la que propiamente es un ser social es la capacidad de comunicación y diálogo: "Esto se ve con claridad meridiana en el hecho de que es propio del hombre el hablar, por medio del cual una persona puede comunicar totalmente a otra sus ideas" (Contra Gentes., lib. 1). Por lo tanto, el hombre es el más comunicativo y a través del lenguaje expresa lo que siente, piensa y crea comunidad. Sin la capacidad de comunicación se hace difícil construir fines comunes como la paz, la justicia o el bien común. Para el maestro Tomás de Aquino, el hombre es un ser perfectible: "Persona significa lo que en toda naturaleza es perfectísimo, es decir, lo que subsiste en la naturaleza racional (Summa Theol., I, q. 29 art. 3). La educación es el medio, por ella se conduce y promociona a la persona al estado perfecto que es la virtud (libre, autónomo, responsable). Para el Aquinate la educación es: "condución y promoción del hombre en cuanto hombre que es el estado de virtud" (In Sent., lib. IV d. 26 q. 1 art. 1)

En esta perspectiva tomasiana del hombre como un ser indidivual y social, fray Sedano desarrolla su concepción de perso- 
na como un ser autodeterminado y comprometido, es decir, que subsiste por sí mismo, pero al mismo tiempo está abierto a los demás. De modo que: "En la precisa medida de su subsistencia e inconfusión, está abierto a lo otro y a los otros ("ese ad")" (Sedano, 2017, p. 86). De esta manera, en la medida en que más se conoce la persona estará más abierta al otro. Pues no hay apertura a los demás sin un conocimiento personal (Sedano, 2017, p.86).

Por lo tanto, no se trata de un personalismo encerrado en sí mismo, individual o narcisista, sino responsable: autónomo y heterónomo. Pues el hombre es responsable de sí mismo, pero se afirma en comunión con lo otro, con los otros y con el Otro. En el encuentro interpersonal es donde se consolida la capacidad de pregunta y de respuesta, es decir, se fortalece la personalidad en comunión, encuentro y diálogo con los demás. Es un proceso cultural de preguntas personales y comunitarias que se trasmiten y van construyendo nuevas formas de concebir y afrontar la realidad. La respuesta no es ajena sino personal y comprometida: con la realidad y medio o contexto en el que nos encontramos; con el prójimo en una actitud de servicio y co-responsabilidad; y con Dios vivo y presente en la historia de la humanidad y en cada acontecimiento (Sedano, 2017, p. 87).

\subsection{Fundamento espistemológico de la pedagogía de la respuesta}

¿Cómo aprende el hombre? El aprendizaje humano es un proceso de formación y desarrollo integral, es decir, de todas las facultades de la naturaleza humana. De modo que, no se educa una parte de la persona sino su totalidad. De la misma manera, el que aprende es el hombre, como una realidad total, cuando desarrolla y perfecciona sus facultades haciéndolas pasar de la potencia al acto. La enseñanza no se reduce al desarrollo de una sola facultad o dimensión de la persona sino de toda ella. En fray Sedano el aprendizaje se caracteriza por ser formador de la persona y de su capacidad de respuesta. 
Para nuestro autor y en seguimiento del personismo tomasiano, toda persona es una realidad siempre en potencia tanto pasiva como activa, es decir, en disposición para recibir como para producir. A este proceso se llama educare y educere o mejor conocido como educabildad. Pero lo propio del ser persona es, ser dueña de sí misma, es decir, autónoma o libre, que no dependa de la voluntad de otro (Sedano, 2017, p. 19). Además de la formación de la persona para el desarrollo y perfeccionamiento de las facultades humanas, en el pensamiento pedagógico del dominico colombiano, es fundamental la formación de la persona para la respuesta. Pero no es una respuesta mecánica o automática a un estímulo sensible externo, sino que es una respuesta que surge desde el interior de la persona, por la que es dueña de sus actos, de sí mimo y puede responder por sí mismo y no por otro o en otro (Sedano, 2017, p. 52).

Para fray Sedano, la formación de la persona y su capacidad de respuesta es un proceso que tiene diversas etapas. En este proceso de aprendizaje epistemológico se sigue los pasos de la "quaestio" como método filosófico. La pregunta es el primer paso de este proceso epistemológico. Esta se caracteriza por ser personal y cuyo fin es la búsqueda de la verdad. Es una pregunta que germina desde el interior de la persona y su fruto es la verdad buscada y encontrada. Sin pregunta no hay respuesta, porque es una pregunta existencial, es decir, sobre problemas reales, y sobre lo que no puedo responder de manera sencilla, sin compromiso sino con una respuesta comprometida.

Según fray Sedano (2017):

En primer lugar, una pregunta ("utrum"), no recibida, sin más, de preocupaciones ajenas, sino suscitada por una inquietud propia y entrañable; por consiguiente, una pregunta viva y existencial, tan vivida y tan vívida que crea la necesidad de búsqueda de la verdad y de una respuesta igualmente viva y existencial (p. 99).

El segundo paso del proceso es la escucha y el conocimiento de las diversas y a veces opuestas opiniones que han surgido en 
torno a un tema en particular y que ha sido cuestionada con una pregunta. Por lo tanto, es un momento de discernimiento de lo que se ha dicho hasta el momento acerca de un tema real. Con una actitud abierta a la escucha, de diálogo y de crítica, hay que contar con la opinión de los demás para la construcción de la verdad y con ella de la respuesta. Pero la respuesta es propia y no ajena a las preguntas personales o comunitarias que han surgido desde el interior de la persona. De modo que, para fray Sedano (2017):

En segundo lugar, laboriosa búsqueda, a veces tanteada de la verdad, en el pensamiento y en la experiencia ajena, no para quedarse ahí, perezosamente ahí, sino como un primer momento para desbrozar caminos hacía la propia y personal respuesta. Etapa en la didáctica medieval, estereotipada bajo la fórmula "Sic et Non" (p. 100).

El tercer paso del proceso es la respuesta personal o personalizada a la pregunta inicial planteada. La respuesta es fruto de discernimiento, de escucha de las diversas opiniones, de diálogo y de síntesis personal. Pero es una respuesta que se caracteriza por ser propia, y no ajena, es decir, libre o autónoma; por aportar nuevos horizontes de comprensión del problema presentado o planteado; y por ser susceptible de análisis, es decir, abierta a seguir construyéndose por ser dinámica y progresiva. De acuerdo con fray Sedano (2017):

La búsqueda no puede quedarse en la sola escucha o en el diálogo. Es preciso que yo mismo, por mi propia cuenta o conducido por otros, comprenda el recorrido alrededor de la realidad piramidal para darme cuenta experiencialmente de sus diversos aspectos o dimensiones. Analizarla, por consiguiente, en sus diversos lados y elementos, movimiento y relaciones con sus coherencias y contrariedades. Análisis ciertamente, en orden a una síntesis, a un juicio personal, a mi "Respondeo dicendum"; verdadera respuesta a mi problema personal o personalizado ( $p$. 100-101). 
Finalmente, el cuarto paso es la crítica en el que se analiza y evalúan las diversas opiniones. La finalidad en este momento psicológico o espistemológico es encontrar lo que hay de verdad en las diversas opiniones y separarla del error.

Con esta experiencia podremos comprender y apreciar el pensamiento ajeno, sin renunciar a juzgarlo con entera libertad y armados de este criterio: descubrir el error en las diversas posiciones, buscar en ellas la verdad oculta en sus unilateralidades y exageraciones, y aceptar de contrapartida, el derecho de los otros a juzgar nuestros puntos de vista (2017, p. 1001).

Por consiguiente, es un proceso epistemológico que se caracteriza por su realismo, porque tiene como punto de partida la realidad; también por el diálogo, porque tiene presente las diferentes opiniones; y por la respuesta, porque se asume una postura personal frente a una realidad o problema concreto.

\subsection{Fundamento metodológico de la pedagogía de la res- puesta}

¿De qué manera se debe enseñar? Fray Sedano incorpora a su propuesta pedagógica el método filosófico de la quaestio de Santo Tomás de Aquino. Con ello, el método de la "quaestio" responde a los intereses de la formación de la persona para la respuesta, el pensamiento crítico y constructivo. Para fray Sedano (1970):

Es cierto que la adopción de este método a base de problematizar, además de ser un riesgo, supone una mentalidad pedagógica. Porque si nuestra pedagogía sigue la trayectoria de un quietismo de la naturaleza, para el que la mente humana es de tal modo pasiva que todo lo recibe de fuera, entonces ideas, criterios, hábitos, orientación no se discute: se imponen (p. 63)

El método de la "quaestio" tiene unos procedimientos que se integran y niveles de conocimientos que llevan finalmente a la cons- 
trucción de la verdad. La primera etapa del procedimiento es el utrum con el que se suscita el problema. La palabra en latín utrum es traducida como: es o no es. La formación de la persona a través del proceso de enseñanza y aprendizaje, orientado a poner en acto las facultades humanas inicia con un planteamiento inquiriente de la verdad (Sedano, 1970, p. 63). En la primera etapa del método se plantea una pregunta de investigación sobre un problema suscitado en el interior de la persona o de la comunidad. Una pregunta que exige una respuesta contundente. El pensamiento crítico inicia con una pregunta problematizadora (Sedano, 1970, p. 64).

La segunda etapa de la quaestio es el videtur y es traducido como: parece que... el "videtur" es la selección de lo que el entendimiento capta a primera vista acerca del tema abordado, a partir de la experiencia y de lo que en general parece más o menos notorio. Por lo tanto, no se trata de una mención inopinada de cualquier percepción, sino contundentes que surgen de la experiencia personal y del diálogo con otras personas. De modo que, la verdad se construye en diálogo y no imponiendo ideas. Para tejer la verdad con la semilla que de ella hay en las opiniones diversas es importante asumir una actitud de escucha y discernimiento (Sedano, 1970, p. 65).

La tercera etapa metodológica es el Sed contra que significa o se traduce como: en cambio, por otra parte. En el método de la "quaestio" el "sed contra" no desempeña una función ni argumentativa ni demostrativa. Es más bien el peso de una autoridad que sobre el tema ha opinado de manera diferente. El sed contra obliga a contrastar lo discutido en las objeciones e introduce una luz nueva, introductoria como preámbulo par el corpus artículi o cuerpo del artículo (Sedano, 1970, p. 69)

La cuarta etapa metodológica es respondeo dicendum... significa o se traduce como: en cambio o solución. Es el corpus o médula del artículo, el núcleo de articulación. Además, es el centro de todo el planteamiento hacía el cual derivó la argumentación de las objeciones (Sedano, 1970, p. 70). En esta etapa del método quaestio es la respuesta personal a la pregunta problematizadora que se 
ha planteado inicialmente. Es un momento de madurez intelectual para responder de sí mismo y por sí mismo después de haber escuchado, dialogado y discernido. De modo que, se trata de un paso del pensamiento critico al constructivista y sintético.

La quinta y última etapa de la metodología es ad primum, ad secundum..., y significa o traduce como: soluciones o respuestas a las abjeciones. Las respuestas a las objeciones surgen de la trabazón demostrativa del "corpus", cuya verdad se revierte (Sedano, 1970, p. 73). Para fray Sedano, el método de la "quaestio" tiene una estructura semejante en la manera como conocemos. Es un método que se caracteriza, como en la manera de conocer, por su realismo (Sedano, 2019, p. 73).

\section{La teoría pedagógica de la pedagogía de la respuesta}

La teoría pedagógica de la pedagogía de la respuesta se fundamenta en la teoría antropológica, epistemológico y metodológica que se orienta a formar al hombre para la respuesta. Además, tiene una fundamentación en la antropología teológica y estructura moral del hombre según el pensamiento tomista. Para Tomás de Aquino: "Cuando decimos que el hombre ha sido hecho a imagen de Dios, entendemos por imagen como dice el Damaceno, un ser dotado de inteligencia, libre albedrío y dominio de sus propios actos" (Summa Theol., I-II Pról.). En efecto, el hombre se asemeja a Dios por el alma cuyas facultades propias y distintivas son la inteligencia y la voluntad. En la medida que desarrollemos las potencias propias del alma y se va perfeccionando y se asemeja o se acerca más a Dios.

Del mismo modo para el Aquinate:

De entre las acciones que el hombre realiza, sólo pueden considerarse propiamente humanas aquellas que son propias del hombre en cuanto que es hombre. El hombre 
se diferencia de las creaturas irracionales en que es dueño de sus actos. Por eso, sólo aquellas acciones de las que el hombre es dueño pueden llamarse propiamente humanas. El hombre es dueño de sus actos mediante la razón y la voluntad; así, se define el libre albedrío como facultad de la voluntad y de la razón. Llamamos, por tanto, acciones propiamente humanas a las que proceden de una voluntad deliberada. Las demás acciones que se atribuyen al hombre pueden llamarse del hombre, pero no propiamente humanas, pues no pertenecen al hombre en cuanto que es hombre (Summa Theol., I-II q. 1 art. 1)

Sobre esta consideración antropológica y moral del pensamiento tomasiano, se pueden resaltar dos aspectos: a). El hombre dueño de sus actos. El hombre es un ser capaz de responder por sí mismo porque es dueño de sus actos, mediante las facultades propias del hombre: la razón y la voluntad y por las que se asemeja a Dios. El objeto de la razón es la verdad y de la voluntad es el bien. De manera que, el desarrollo y perfeccionamiento de éstas hace que el hombre actúe por sí mismo, con autonomía y responsabilidad. b). Las acciones propiamente humanas, es decir, aquellas de las que el hombre es dueño porque proceden de sus propias facultades. Se trata de actos libres, es decir, de actos morales y humanos. De esto se desprende el humanismo del hombre, distinguido de las creaturas irracionales por ser dueño de sus actos mediante las facultades de la razón y de la voluntad, y porque sus acciones surgen de estas dos facultades se llaman con propiedad acciones humanas.

Desde esta perspectiva tomasiana del hombre fray Sedano se sirve para desarrollar su pedagogía de la respuesta y resaltar que el hombre se hace en la medida en que pone en acto sus potencias, porque las desarrolla y perfecciona y por eso hace el llamado de: "ser en plenitud lo que eres en germen" (2017, p. 156).

Además, fray Sedano incorpora algunos aspectos de la formación humana del maestro Blanchet a su propuesta pedagógica. De modo que encontramos algunos rasgos evidentes en la concepción de hombre en su libertad (Blanchet, 1974, pp. 9-10), así como el de 
germen o semilla (Blanchet, 1974, p. 15). La pedagogía de la respuesta no estuvo desligada de la vida personal de fray Sedano. El fraile dominico y formador siempre se mostró con un espíritu libre, renovador y progresista. De modo que la pedagogía de la respuesta ocupa en primer lugar una categoría experiencial que revela el itinerario progresivo de construcción existencial de su autor (Argüello, 2019, p. 53). Es un proyecto de formación integral centrado en la persona al ser formando en la inteligencia y la voluntad, para el pensamiento crítico, la libertad responsable y comprometida.

\section{La pedagogía de la respuesta como modelo pedagógico}

La teoría pedagógica da origen al modelo pedagógico, que está constituido por los componentes curriculares. De esta manera, "un currículo es, pues, la caracterización de los propósitos, los contenidos, la secuenciación, el método y la evaluación. Cada uno de estos elementos resuelve una pregunta diferente, pero interrelacionada" (De Zubiría. 2015, p. 40). Las preguntas y componentes del currículo son las siguientes: ¿Para qué enseñar? Propósitos educativos; ¿Qué enseñar? Contenidos; ¿Cuándo enseñarlo? Secuenciación; ¿Cómo enseñarlo? Metodología; ¿Con qué enseñarlo? Recursos didácticos; ¿Se cumplió o se está cumpliendo? Evaluación. De modo que la pedagogía de la respuesta como modelo pedagógico debe reunir las caracterizaciones del currículo.

\subsection{El primer componente de la pedagogía de la respuesta: Propósitos.}

La primera pregunta y fundamental a la que debe responder la pedagogía de la respuesta es: ¿Para qué enseñamos? Y se refiere al componente del propósito o finalidad del acto educativo. La finalidad de la pedagogía de la respuesta consiste en poner en acto las potencias humanas para decidirse. El hombre es una totalidad, pero siempre en potencia, que debe actualizarse por medio de la 
educación para responder de manera libre, es decir, autónoma y responsable y con lo anterior está incoada la capacidad y el acto de decidir. De esta manera, cuando se responde se decide.

Para fray Sedano, responder en su radical sentido es: tener la "capacidad de responder al compromiso asumido y exigencia de respuesta en virtud de la palabra empeñada; palabra de fidelidad comprometida y comprometedora" (2017, p. 32). Cuando la persona responde, es más que una actitud o una palabra, es la persona misma. Pero, formar en la respuesta es finalmente un proceso en el que debe ser formada o educada la persona. Nuestro autor Ilama cultivarse a este proceso: "Cultivarme para la respuesta. Es la exigencia primordial. Porque no nací aprendido. Ni porque la respuesta va a brotar, porque sí, como por arte de magia" (Sedano, 2017, p. 52). El propósito teleológico de la pedagogía de la respuesta es formar a la persona para la capacidad de decidirse. Así, según fray Sedano (2017): "aprendemos a responder, respondiendo. $Y$ responder, es decir, comprometerse es, al fin, decidirse" (p. 97). Por lo tanto, la formación es un proceso integral e intencional que comienza con el cultivo de la persona para la respuesta, el compromiso y, finalmente, para la capacidad de decidir.

El hombre actua de manera libre y en a cada acto libre está eligiendo o decidiendo. Lo propio del hombre es la capacidad de decisión por la que construye su proyecto de vida. Para fray Sedano (2017): "Decidirse es el acto central de nuestra libertad. Tan central y definitivo que es el responsable de tejer la trama de nuestra historia humana. Sin él no hacemos historia: nos la hacen" (p. 97). Por lo tanto, la formación está centrada en la persona, que debe ser conducida y promovida para que decida por sí mismo y no por otro (Sedano, 2017, p. 99).

\subsection{El segundo componente de la pedagogía de la respuesta: Contenidos.}

La segunda pregunta que debe responder la pedagogía de la respuesta es: ¿Qué enseñar? Y se refiere al componente de los con- 
tenidos y está integrado por los temas selecionados, su carácter e importancia. La pedagogía de la respuesta se centra en la formación de la persona, en el desarrollo y perfeccionamiento de todas sus facultades para la capacidad de decidir. Pero, la persona cultiva y desarrolla sus facultades en la medida que responde de sí mismo por sí mismo. Al decir de fray Sedano: "Porque de eso se trata, dar mi propia respuesta. En ello me va la vida. Está en juego mi realización personal, mi felicidad, mi plenitud" (2017, p.37). El proceso o cultivo para la respuesta y para la desición es planteada por fray Sedano de manera gradual de la siguiente manera:

No puedo realizarme sin responsabilizarme; no puedo responsabilizarme sin personalizarme; no puedo responsabilizarme sin definirme; no puedo definirme sin autodeterminarme; no puedo autodeterminarme sin comprometerme; no puedo comprometerme sin decidirme (2017, p. 38).

El proceso de formación es intencional en cuanto se centra en la persona y en la capacidad de decidir. Además, es un proceso intencional que se caracteriza por ser dinámico al poner en acto y desarrollar de manera armónica las diferentes facultades humanas como las dimensiones de la persona. Del mismo modo, involucra en el proceso de formación persona y comunidad, responsabilidad y corresponsabilidad (Sedano, 2017, p. 38).

\subsection{El tercer componente de la pedagogía de la respuesta: secuenciación.}

La tercera pregunta que debe responder la pedagogía de la respuesta es: ¿Cuándo enseñarlo? Y se refiere al componente de la secuenciación. De modo que, deben fijarse los criterios generales para la secuenciación de las intenciones educativas. La pedagogía de la respuesta se centra en el desarrollo de las facultades humanas para la respuesta desde lo personal a lo social. En la pedagogía de la respuesta como modelo pedagógico da primacía a la persona: porque es el centro de formación, porque la autonomía o el ser 
dueño de sus actos o responsable define respectivamente al hombre. La semilla de humanidad que está en potencia por sí misma va tomando fuerza a través de sus acciones (Sedano, 2017, p. 132).

La semilla de humanidad que está en potencia requiere para su crecimiento que pueda ser ayudada por su semejante porque, "Cada alumno es un sujeto autónomo, pero no es un sujeto puro: incrustado en colectividades, sólo se autoafirma y desarrolla sus potencialidades por la mediación de la interación social" (1975, p. 106). Para Sedano no puedo autodeterminarme sin comprometerme. Realizarse es corresponder, es decir, comprometerme con el otro (Sedano, 2017, p. 87).

\subsection{El cuarto componente de la pedagogía de la respuesta: Metodología.}

La cuarta pregunta que debe responder la pedagogía de la respuesta es: ¿Cómo enseñar? Y se refiere al componente de la metodología. Este componente toca a la realación existente entre maestro, conocimiento y alumno. Para fray Sedano la metodología, enm el marco de la virtud de la estudiosidad, está orientada a la búsqueda de la verdad y debe estar integrada por los pasos de análisis, síntesis, y crítica. (2000, pp. 124-125)

El primer paso para una enseñanza significativa es la probelmatización de la realidad a través de una pregunta. Ella nos pone en contacto con las cosas y nos abre a la búsqueda de las semejanzas y desemejanzas de las diversas experiencias. El segundo momento de la enseñanza es la comprensión de la realidad como un todo. Es importante sintetizar las diversas opiniones. El tercer momento de la enseñanza, es la crítica de las diversas opiniones como a nuestra propia síntesis, siempre en contraste con la realidad (Cárdenas, 2013, p. 31) 


\subsection{El quinto componente de la pedagogía de la respuesta: Recursos didácticos.}

La quinta pregunta que debe responder la pedagogía de la respuesta es: ¿Cómo enseñar? Se trata del componente de la didáctica o del arte de enseñar. Para la pedagogía de la respuesta, en el proceso de enseñanza y aprendizaje, es fundamental la palabra humana. De modo que el recurso humano e instrumental es la palabra por la que comunicamos nuestras ideas y por la que dialogamos para la construcción de la verdad. La palabra expresa lo que somos y pensamos. La enseñanza es conducir y promover a la persona para que responda por sí mismo. Desde la perspectiva de la pedagogía de la respuesta somos la respuesta que damos.

No se trata de cualquier tipo de palabra, sino de la palabra humana y humanizadora. La palabra humana mal usada llega a deshumanizar. Por lo tanto, se refiere a una palabra creadora, capaz de producir vida. De manera que, no se trata de una palabra que transmite saberes o conocimientos sino de una palabra que soy yo: una realidad única entre mi intimidad y la palabra expresada. En consideración de la experiencia sajoniana: "Mi palabra debe ser respuesta o pregunta, no mensaje de salvación: obrar así es abordar al otro con intenciones, prejuicios, es tomarlo como una materia prima susceptible de ser manipulada en función de mi cosmovisión" (1957, p. 107).

\subsection{Sexto componente de la pedagogía de la respuesta: evaluación.}

La sexta pregunta que debe responder la pedagogía de la respuesta es: ¿Qué, cómo y cuándo evaluar? Y se refiere al componente de la evalución. Efectivamente no se evalúan contenidos sino la capacidad de respuesta de la persona. Es una evaluación de la vida de manera procesasual. Según fray Sedano: "Y, entonces, escogiste tu vida como un don y una tarea, como una gracia y una conquista, como una semilla que urgía tu cultivo; en suma, como una pregunta en espera de tu respuesta". (2017, p. 130). 
El actuar es propio de la persona. El hombre se distingue de las demás criaturas por ser dueño de sus actos mediante la voluntad y la razón. Dos potencias propias del hombre cuya fuerza interior hace el acto propiamente humano. Abdicar a la propia respuesta es el pecado del hombre. Fray Sedano nos recuerda y escenifica el pecado de Adán, del primer hombre descrito en la Biblia, y pone el acento no tanto en la soberbia del hombre de querer ser como dioses, sino en no ser capaz de responder por sí mismo, pues el hombre culpa a la mujer y esta a la serpiente.

Toda persona corre el riesgo de escenificar el pecado abdicando a su propia respuesta, cuando teniendo conciencia de sí mismo y pensando por sí mismo deja que otros piensen por él y respondan por él. Por el contrario, puede llegar a una existencia en crecimiento cuando es capaz de decidir por sí mismo y ser dueño de sus actos, además promovida en su humanidad en la tarea humanizadora de quienes lo rodean.

\section{Hagamos al ser humano: Hacia un modelo de pedagogía humanista.}

La pedagogía es una reflexión sobre el acto formativo del hombre. Dios es el primer pedagogo de la humanidad, desde el origen como se afirma en el libro del Génesis: "Dijo Dios: hagamos al ser humano..." (Gn. 1, 26). La pedagogía humana es un acto formativo del ser humano en el que se continúa la obra originaria de Dios, hacedora de humanidad. De ahí la afirmación de fray Sedano (2017): "La pedagogía humana es sencillamente una colaboración de la pedagogía de Dios, que promueve integralmente el dinamismo de nuestra autorealización humana, para tomar nosotros, solamente nosotros, las riendas de nuestro propio destino" (p. 165). De esta manera, la pedagogía humana es una pedagogía continuada, cuyo objeto es el desarrollo y actualización de todas las capacidades propias del hombre por las que se asemeja a su hacedor. 
Según Tomás de Aquino, "A Dios se asemejan las cosas, en primer lugar, y de un modo muy común, en cuanto que existen; en segundo lugar, en cuanto que viven, $y$, finalmente, en cuanto que saben o entienden" (Summa Theol., I q. 93 art 2). De igual modo, para el Aquinate, en el hombre la imagen se encuentra principalmente en el alma y en el cuerpo como vestigio: "Así, pues, en el hombre se encuentra la semejanza de Dios como imagen en cuanto a la mente y como vestigio en sus otras partes" (S. Th. I q. 93 art. 6). Y así la formación humana es crecimiento, como lo manifiesta la tomista Edith Stein (2008): "Dios ha dado al hombre su disposición natural y se la ha dado en forma de una semilla que está determinada al desarrollo y a la evolución" (p. 192). Así, la pedagogía es un hacer crecer al hombre desde lo interior a lo exterior en la medida que actualizamos las potencias del alma y del cuerpo de manera armónica e integral. El hombre es siempre un ser perfectible.

El Aquinate distingue en las operaciones humanas tres dimensiones o líneas de actividad: la especulación, la acción y la producción. Pero en ellas establece una doble distinción desde la categoría hacer para los que utiliza en latín las palabras agere y facere. El "agere" se refiere al acto inmanente que permanece en el mismo sujeto; el "facere" se refiere al acto transeúnte que pasa a la materia. De modo que, el resultado del "agere" es la perfección del agente; en el del "facere" es la perfección del artefacto. Si la pedagogía es un hacer al hombre, nos podemos preguntar: ¿El acto educativo es un "agere" o un "facere"? Es un "agere", ésta sería la respuesta, desde el proceso de la enseñanza y del aprendizaje, por parte del docente y discente. Por parte del maestro se requiere saber o tener conocimiento de lo que enseña, conducción y promoción del humanismo del discente; por parte del alumno se necesita docilidad, desarrollo y perfeccionamiento de su personalidad desde la virtud. Se trata de un proceso de formación y transformación: "El niño, el sujeto de la educación, tiene para el educador un doble aspecto: es nuevo en un mundo que le es extraño y está en proceso de transformación, es un nuevo ser humano y se está convirtiendo en un ser humano" (Arendt, 2016, p. 285). 
Tomás de Aquino es un teólogo por vocació, pero como teólogo no excluye de su pensamiento los problemas del hombre. La mayor parte de su vida académica la pasó investigando, escribiendo y enseñando. Como pedagogo se preocupó por la formación del hombre, de manera especial por la formación humanista. Cuando Tomás de Aquino trata la condición humana acentúa sobre la formación humanista, es decir, sobre la formación moral del hombre. Para el Aquinate, la formación humanista o moral del hombre se desarrolla a través de las virtudes. Entre las virtudes dintinguió las morales, intelectuales y artísticas. La perfección teórica (virtudes intelectuales) está en la verdad conocida, la perfección fáctica (virtudes prácticas) en la obra realizada, la perfección moral (virtudes cardinales) está en hacer bueno al hombre. Pero, solo las virtudes morales hacen bueno al hombre y a su acto.

Tomás de Aquino se interesó por las virtudes morales, las que "hacen" al hombre, verdaderamente hombre, sin la cual no hay plenitud de lo humano. Según el Aquinate: "Por esta razón es preciso que la virtud de una cosa se defina por orden al bien. Por consiguiente, la virtud humana, que es un hábito operativo, es un hábito bueno y principio operativo del bien" (Summa Theol., I-II q. 55 art. 3). Del mismo modo, afirma: "El vivir se toma en dos sentidos. Algunas veces significa la existencia del viviente, y así pertenece a la esencia del alma, que para el viviente es principio de su existencia. Otra significa la actividad del viviente, y así vivimos rectamente por la virtud, en cuanto que por ella llevamos rectamente a cabo nuestras operaciones" (Summa Theol., I-II q. 56 art. 1 ad 1). De ahí que, no toda virtud es virtud moral sino aquella que hace bueno al sujeto y a sus actos (Cf. S.Th. I-II q. 58 art. 1).

El concepto de virtud es fundamental como su desarrollo en la vida del hombre para su formación humanista. Son diferentes las concepciones de virtud que hay en el pensamiento del Aquinate, entre ellas retoma la definición de Aristóteles: "La virtud es la que hace bueno a su poseedor y buena su obra" (Summa Theol., I-II q. 55 art. 3 Sec., cont.), o la elaborada por el mismo Santo Tomás: "Virtud es una buena cualidad de la mente por la que se vive con rectitud, de la cual nadie hace mal uso, y que Dios obra en noso- 
tros sin nosotros" (Summa Theol., I-II q. 55 art. 4). Por tanto, para Santo Tomás: "la virtud humana es un hábito que perfecciona al hombre para obrar bien" (Summa Theol., I-II q. 58 art. 3). En suma, para él la formación humanista a través de las virtudes morales están en primer lugar a la formación profesional, es decir, intelectual y técnica (S. Th. I-II, q. 57 art. 3 ad. 2).

De manera forma, cuando el Aquinate trata lo concerniente a la formación de la persona en lo referente a una formación profesional (intelectual, técnica) y humanista, opta por la formación humanista del hombre. Y esto se debe a que solo las virtudes morales son las que hacen al hombre. De esta manera, hacer al hombre, pedagógicamente, es coayuvar en el desarrollo, crecimiento y actualización de las potencias o facultades humanas, desde las virtudes morales que hacen bueno al hombre y a sus actos. Pero, en la educación actual se derime entre la formación profesional de carácter científico, técnico e innovador y la humanista. Sin embargo, gana más terreno en el ámbito educativo la formación profesional sobre la humanista.

La educación de la persona es fundamental para el desarrollo y progreso de un país. Ahora se tiene más consciencia de su importancia para la reducción de la pobreza y fomentar el desarrollo sostenible. Pero en la educación actual hay una tendencia a creer que la educación de calidad depende de la inversión que se haga en ciencia, tecnología e innovación. Esta idea o impresión la ha recogido Oppenheimer (2015, p. 292) en uno de sus recorridos por diferentes paises del mundo y ha llegado a la conclusión que las claves del éxito en el siglo XXI, son la innovación y la creatividad como pilares del progreso en lugar de las humanidades.

No obstante, hay una serie de pensadores que han realizado reflexiones de tendencia humanista y han Ilamado la atención sobre la importancia de las humanidades en los centros educativos para la formación humanista del hombre. Es importante aclarar que esto se debe al poco espacio que tienen y han venido perdiendo las humanidades en las intituciones educativas en todos sus niveles y que se evidencia en el PEI, Programas de Formación 
o Pensum Académico. Aunque las humanidades y el humanismo no son de horas ni de espacios institucionalizados, es de toda la vida. Pero, es importantes que hagan parte o tenga su lugar dentro programación académica de las instituciones educativas. Además, no se trata de separar la formación humanista de la formación profesional sino de priorizar e integrar. En este sentido, la prioridad deben tenerla las humanidades en la formación del hombre (OPPENHEIMER, 2018, p. 2016).

De esta manera, para la filósofa Martha Nussbaum (2016, p. 189, preocupada por el grado en que el estudio de las humanidades está siendo abandonado en beneficio de aprendizajes más directamente vinculados con las actividades económicas, con el ánimo de obtener beneficios y en detrimento de la educación para la ciudadanía). Del mismo modo, el filósofo Fernando Savater (2013, p. 114), considera que, entre las grandes y diferentes inquietudes de nuestro tiempo, se llega a la conclusión de que todas ellas deben afrontarse desde la escuela o a educación. Además, señala lo fundamental de las humanidades en los planes de estudios para el desarrollo pleno del humanismo del hombre. Las empresas deben encaminarse hacia el valor de lo humano por encima de la producción material. La calidad de un producto debe pasar primer por la calidad humana e interpersonales de quienes lo hacen posible. De este modo, Carlos Raul Yepes (2017, p. 85)., expresidente de Bancolombia, después de varios años como empresario, resalta el valor de lo humano y la importancia del otro

Lo propio del ser humano es su humanidad. El objeto de la pedagogía humanista es hacer al hombre plenamente hombre, es decir, cultivar su humanidad: hacer crecer lo humano del hombre, mediante el desarrollo de las potencias del hombre, del alma y del cuerpo, haciéndolas pasar de la potencia al acto. Además, implica formar al hombre para el encuentro y reconocimiento del otro, de formar al hombre para ser ciudadano del mundo y del mundo globalizado. Una de las competencias a desarrollar en la persona y en el marco de los retos y desafíos de la educación actual y futura, es la interculturaldiad y ciudadanía del mundo, es decir, la ciudadanía cosmopolita. Esto conlleva un conocimiento 
propio y un reconocimiento del otro, el conocer las propias raíces culturales y reconocer que pertenece a un universo cultural con el que debe dialogar, escuchar y respetar desde las virtudes sociales de la verdad, amor y justicia. De modo que: "Podríamos decir, por tanto, que el reconocimiento de la ciudadanía social es conditio sine qua non en la construcción de una ciudadanía cosmopolita que, por ser justa, haga sentirse y saberse a todos los hombres ciudadanos del mundo" (Cortina, 2 017, p. 222). No es una competencia fácil a desarrollar en una sociedad que tiende al individualismo y al narcisismo y donde el otro es un extraño llamando a la puerta (Bauman, 2016). Como muy bien lo expresa Nussbaum (2017): "Es relativamente fácil construir una educación señorial para una élite homogénea. Es mucho más difícil preparar a la gente de orígenes muy diversos para una compleja ciudadanía universal" (p. 321).

El maestro Tomás de Aquino ha considerado en su pensamiento que "el hombre es un amigo para el hombre" (Summa Theol., I-II q. 28 art. 1). El doctor de la humanidad ha sido un filántropo. Su amor por la humanidad hizo que mantuviera una actitud de diálogo con otras culturas diferentes en la forma de pensar y creer. En efecto, es importante desarrollar o potencializar la mente respetuosa que sea capaz de observar, aceptar y comprender a esos otros y procurar trabajar con ellos de manera efectiva (Gardner, 2016, p. 16). "Hoy perdemos cada vez más la capacidad de escuchar. Lo que hace difícil escuchar es sobre todo la creciente focalización en el ego, el progresivo narcisismo de la sociedad" (Han, 2017, p. 113). Desde la pedagogoía humanista es importante el crear una cultura del encuentro como lo enuncia actualmente el papa Francisco. El homo viator es el que crea puentes que unen y no murallas que separan: "El caminar tal vez se encuentre a la base de la cultura del encuentro. Los hombres se encuentran, se comunican" (papa Francisco, 2018, p. 22). De modo que, las humanidades seguirán siendo central es en la formación de la persona y aún más en la era de la información y de la automatización. 


\section{Conclusión}

La Pedagogía de la Respuesta es una propuesta educativa de fray de Jesús Sedano, quien ha reflexionado sobre la formación de la persona desde un enfoque dominicano y tomista. Además, su propuesta pedagógica es fruto de la experiencia como formador de una generación de religiosos y laicos dominicos. En el ámbito educativo son varios los escritos e investigaciones que han tenido presente la obra de fray Sedano. La Pedagogía de la Respuesta se centra en la formación de la persona para la responsabilidad, autonomía y libre, es decir, en la capacidad de decidir por sí mismo. Los diferentes escritos educativos o pedagógicos cuando citan la pedagogía de la respuesta se refieren a una educación que tenga como finalidad la formación para la respuesta y de decisión personal. Los modelos pedagógicos existentes son varios y diferentes, todos ellos buscan responde por el tipo de hombre y de sociedad que se quiere contribuir a formar. La Pedagogía de la Respuesta es un modelo pedagógico porque reúne los fundamentos teóricos y los componentes curriculares de todo modelo pedagógico.

La pedagogía de la respuesta tiene un enfoque humanista por inspirarse en el pensamiento tomasiano que se orienta principalmente, a la formación del hombre. La pedagogía humanista es hacer al hombre propiamente hombre a través del desarrollo de sus facultades. El hombre es un ser completo, pero no acabado, es y se hace a través de las virtudes, especialmente de las virtudes morales. Ante una tendencia educativa para la ciencia, técnica e innovación, en informar más que en formar, la pedagogía humanista propone para la educación actual la educación en las virtudes morales porque hacen bueno al hombre y bueno su acto. Lo primero es formar al hombre y luego se informa. De modo que, en los ambiomas educativos lo primero es formar humanamente al hombre y posteriormente informarlo profesionalmente. 


\section{Referencias bibliográficas}

Alvira, R., y Spang K. (2006). Humanidades para el siglo XXI. Pamplona, España: Eunsa.

Aparicio, O.Y. (2018). El constructivismo y el construccionismo. Revista Interamericana de Investigación, Educación y Pedagogía, RIIEP, 11(2). https://orcid.org/0000-0003-3535-6288

Arendt, H. (2016) Entre el pasado y el futuro. Ocho ejercicios sobre la reflexión política. Buenos Aires, Argentina: Ariel.

Azevedo, S. R. J. (2014). La composición histórica de la educación religiosa como componente curricular. Revista Interamericana de Investigación, Educación y Pedagogía, RIIEP, 7(1). DOI: https://doi.org/10.15332/ s1657-107X.2014.0001.03

Bauman, Z. (2016). Extraños Ilamando a la puerta. Barcelona, España: Paidos.

Cortina, Adela. (2017). Ciudadanos del mundo. Hacia una teoría de la ciudadanía. Madrid, España: Alianza.

Han, Byung-C. (2017). La expulsión de lo distinto. Barcelona, España: Herder.

Gardner, H. (2016). Las cinco mentes del futuro. Barcelona, España: Paidos.

Nussbaum, M. C. (2016). Sin fines de lucro. Por qué la democracia necesita de las humanidades. Madrid, España: Katz.

Nussbaum, M. C. (2017). El cultivo de la humanidad. Una defensa clásica de la reforma en la educación liberal. Barcelona: Paidos.

Oppenheimer, A. (2015). iCrear o morir! La esperanza de América Latina y las cinco claves de la INNOVACIÓN. Bogotá, Colombia: Debate. 
Oppenheimer, A. (2018). iSÁLVASE QUIEN PUEDA! El futuro del trabajo en la era de la AUTOMATIZACIÓN. Bogotá, Colombia: Debate.

Papa Francisco. (2018). Política y sociedad. Conversaciones con Dominique Wolton. Madrid, España: Encuentro.

Savater, F. (2013). El valor de educar. Bogotá, Colombia: Planeta.

Sedano, G., J.J. (1970). El método teológico en Santo Tomás de Aquino. Bogotá, Colombia: USTA

Sedano, G., J.J. (2017). Hacia una pedagogía de la respuesta: horizonte tomasiano para la formación integral de la persona. Bogotá, Colombia: USTA.

Stein, Edith. (2003). Escritos antropológicos y pedagógicos. (Obras completas, IV). Burgos, España: Monte Carmelo.

Yepes, C. R. (2017). Por otro camino. De regreso a lo humano. Bogotá, Colombia: Aguilar.

Zubiría, S., J. (2002). Tratado de pedagogía conceptual. Los modelos pedagógicos. Bogotá, Colombia: Fundación Alberto Merani.

Zubiría, S., J. (2015). Los modelos pedagógicos. Hacia una pedagogía dialogante. Bogotá, Colombia: Magisterio. 